\title{
Changes in tau phosphorylation levels in the hippocampus and frontal cortex following chronic stress
}

\author{
C. Yang ${ }^{1}$, X. Guo ${ }^{1}$, G.H. Wang ${ }^{1,2}$, H.L. Wang ${ }^{1}$, Z.C. Liu ${ }^{1}$, H. Liu ${ }^{1}$, Z.X. Zhu ${ }^{1}$ and Y. Li ${ }^{1}$ \\ ${ }^{1}$ Department of Psychiatry, Renmin Hospital, Wuhan University, Wuhan, China \\ ${ }^{2}$ Institute of Neuropsychiatry, Wuhan University, Wuhan, China
}

\begin{abstract}
Studies have indicated that early-life or early-onset depression is associated with a 2- to 4-fold increased risk of developing Alzheimer's disease (AD). In $A D$, aggregation of an abnormally phosphorylated form of the tau protein may be a key pathological event. Tau is known to play a major role in promoting microtubule assembly and stabilization, and in maintaining the normal morphology of neurons. Several studies have reported that stress may induce tau phosphorylation. The main aim of the present study was to investigate possible alterations in the tau protein in the hippocampus and frontal cortex of 32 male Sprague-Dawley rats exposed to chronic unpredictable mild stress (CUMS) and then re-exposed to CUMS to mimic depression and the recurrence of depression, respectively, in humans. We evaluated the effects of CUMS, fluoxetine, and CUMS reexposure on tau and phospho-tau. Our results showed that a single exposure to CUMS caused a significant reduction in sucrose preference, indicating a state of anhedonia. The change in behavior was accompanied by specific alterations in phospho-tau protein levels, but fluoxetine treatment reversed the CUMS-induced impairments. Moreover, changes in sucrose preference and phospho-tau were more pronounced in rats re-exposed to CUMS than in those subjected to a single exposure. Our results suggest that changes in tau phosphorylation may contribute to the link between depression and AD.
\end{abstract}

Key words: Alzheimer's disease; Depression; Tau; Chronic unpredictable mild stress (CUMS); Re-exposure to CUMS; Fluoxetine

\section{Introduction}

Previous studies have shown an association between depression and dementia, and it has been suggested that lifetime depression is associated with a 2- to 4-fold increased risk of developing Alzheimer's disease (AD) (14). For example, a cross-sectional (case-controlled) study suggested that a history of depression, even 25 years prior to $A D$ onset, was significantly associated with an almost 2-fold increase in the likelihood of developing $A D$, and that depression symptoms within the year before the onset of $A D$ were associated with an almost 5-fold increased risk (4).

The association between depression and $A D$ suggested by the epidemiologic data may be partially explained by several biological mechanisms, including alterations in glucocorticoid steroids, hippocampal atrophy, increased deposition of $\beta$-amyloid plaques, inflammatory changes, deficits in nerve growth factors, and altered neuronal plasticity $(1,5,6)$. Aggregation of abnormally phosphorylated tau, the main constituent of neurofibrillary tangles, is most likely a key pathological event of AD (7). Interestingly, research has shown that tangles accumulate in greater numbers in the hippocampus of AD patients with depression than in those without depression $(8,9)$.

Growing evidence indicates that the cytoskeleton, a structural system including microtubules and microtubuleassociated proteins (MAPs), may play an important role in stress-induced impairments of neuronal structural plasticity (10). Accounting for more than $80 \%$ of the MAPs, tau is known to promote microtubule assembly, stabilize existing microtubules, and to maintain the normal morphology of neurons (7). Developmental and functional regulation of phosphorylation modulates the function of tau proteins in controlling microtubule dynamics during normal neurite outgrowth and maturation. Proper regulation of microtubule dynamics (assembly and disassembly) is essential for normal cell morphology, function, and viability. Accumulating evidence suggests that hyperphosphorylated tau sequesters normal MAPs and disrupts

Correspondence: G.H. Wang, Department of Psychiatry, Institute of Neuropsychiatry, Renmin Hospital, Wuhan University, Jiefang Road 238\#, Wuhan 430060, China. Fax: +86-27-8807-2022. E-mail: wghyc2012@126.com

Received June 6, 2013. Accepted October 8, 2013. First published online March 3, 2014. 
microtubule dynamics (7). Furthermore, acute stresses, such as ether exposure, starvation, forced swimming in cold water, or restraint stress, induce tau phosphorylation in mice (11).

Based upon these studies, we speculated that chronic stress in depression could produce an increase in tau phosphorylation similar to that observed after acute stress. Thus, antidepressant drugs could possibly attenuate the impact of depression on development of $A D$. It would be interesting to explore what role tau might play (e.g., whether changes in tau in depression are similar to those in $A D$ and what effects antidepressant drugs have on tau). The primary aim of the present study, therefore, was to investigate changes in tau in the rat hippocampus and frontal cortex following stress, fluoxetine (FLX) treatment, and re-exposure to stress.

It is believed that chronic stress is a crucial factor involved in depression onset and relapse (12). Chronic unpredictable mild stress (CUMS)-induced depression is seen as the most promising and valuable depressive model in animals, and it has been widely used for investigating the pathophysiology of depression and its associated therapeutic interventions (13). Therefore, we used CUMS in our study.

\section{Material and Methods}

\section{Animals}

A total of 32 male Sprague-Dawley rats weighing 200$250 \mathrm{~g}$ were obtained from the Center for Experimental Animals at Wuhan University for use in this experiment. All animals were housed in groups of four to five in plastic cages with a controlled environment, a 12:12-h light-dark cycle, and ad libitum food and water for 1 week before the start of the investigation. The experimental procedures were conducted in conformity with the instructions for the care of laboratory animals issued by the Ministry of Science and Technology of the People's Republic of China in 2006.

\section{FLX treatment}

FLX (ShangHai JinHuan, China) was dissolved in physiological saline immediately before each use. Intraperitoneal injections of FLX (10 mg/kg) (14) or saline vehicle $(V)$ were administered daily to the rats for 3 weeks.

\section{Experimental design}

The animals were separated into one of four groups: Control +V $(n=8), \quad$ CUMS +V $(n=8), \quad$ CUMS + FLX $(n=8)$, or CUMS + FLX + CUMS $(n=8)$. The fourth group included a 1-week drug washout prior to re-exposure to CUMS. Sucrose preference was used to assess potential behavioral abnormalities after CUMS, FLX treatment, and re-exposure to stress. The rats were housed in groups during the experimental period, while the stress conditions, all injections, and behavioral assessments were delivered in isolation. Details of the experimental procedures are given in Figure 1.

\section{CUMS}

The CUMS procedure was adopted with slight modifications from that described by Luo et al. (15), and some stressors (e.g., empty bottle of water, restricted food) were adapted from Moreau et al. (16). The rats were subjected to various stressors for 3 weeks. These included cage tilting $\left(45^{\circ}\right)$ for $24 \mathrm{~h}$, damp sawdust (200 $\mathrm{mL}$ water in a cage) for $24 \mathrm{~h}$, predator sounds (recording of an adult cat for $15 \mathrm{~min}$ ), swimming in $25^{\circ} \mathrm{C}$ water for $15 \mathrm{~min}, 24 \mathrm{~h}$ of food deprivation immediately followed by $1 \mathrm{~h}$ of restricted access to food ( 5 micropellets), $24 \mathrm{~h}$ of water deprivation immediately followed by 1-h exposure to an empty bottle, tail clamping for $1 \mathrm{~min}$, shaking for $15 \mathrm{~min}$ at $120 \mathrm{rpm}$ on a rocking bed, immobilization for $1 \mathrm{~h}$ in a $25 \times 8-\mathrm{cm}$ cylindrical plastic rodent restrainer, and alterations of the light-dark cycle. The animals were exposed to one stressor at a time; stressors were never presented simultaneously.

\section{Sucrose preference test}

In the present study, we used a sucrose preference test to assess anhedonia. All animals were first trained to consume a palatable, weak ( $1 \%)$ sucrose solution. Before each test, the animals were deprived of water for $12 \mathrm{~h}$ (8:00 pm-8:00 am). The rats were presented with a bottle of $1 \%$ sucrose solution and a bottle of water during a $1-\mathrm{h}$ window (8:00-9:00 am) as originally described by Willner et al. (17). The sucrose preference was calculated according to the following formula: sucrose preference $(\%)=($ sucrose intake/total fluid intake $) \times 100 \%$.

\section{Drug washout period}

Although FLX and norfluoxetine have half-lives of several days in humans, in rats the elimination half-lives are approximately 9 and $15 \mathrm{~h}$, respectively (18). Thus, it is likely that tissue concentrations of this selective serotonin reuptake inhibitor (SSRI) fall substantially after 1 day (19). The washout period for FLX is reported to be different in different articles. We adopted 1 week as the washout period in the present study.

\section{Western blot analysis of tau}

At the end of the last test, the animals were anesthetized with $20 \%$ ethylcarbamate $(7 \mathrm{~mL} / \mathrm{kg})$ at room temperature and decapitated. The brain was rapidly separated from the skull, and the hippocampus and frontal cortex were dissected from both hemispheres while the brain was on an ice-chilled plate, and they were then immediately frozen on dry ice and kept at $-80^{\circ} \mathrm{C}$ until analysis.

Western blot analysis was performed as previously reported (20), with slight modifications. Briefly, frozen tissue samples were thawed and homogenized in buffer. Homogenates were cleared by centrifugation $(12,000 \mathrm{~g}$, 


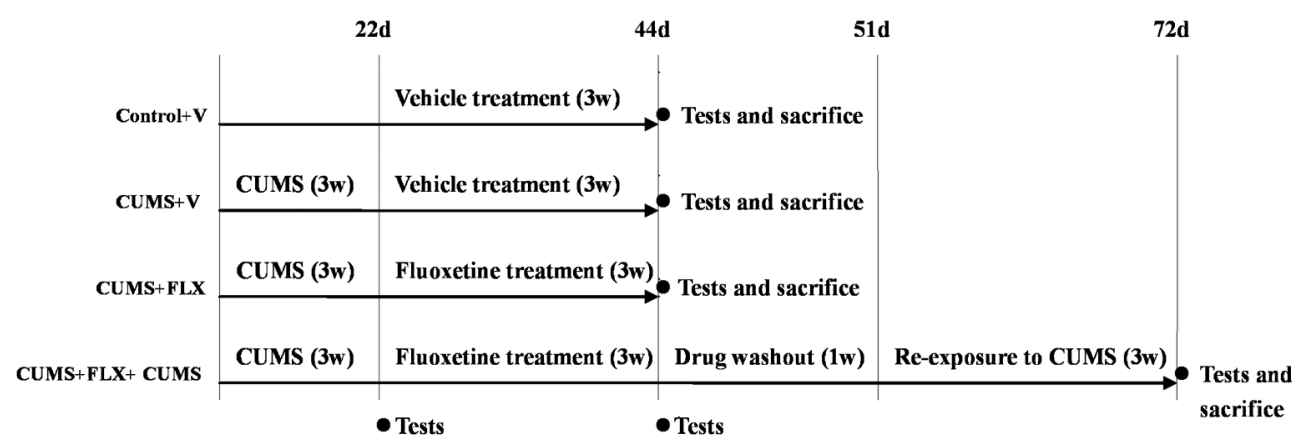

Figure 1. Details of the experimental procedure. Control $+\mathrm{V}, \mathrm{CUMS}+\mathrm{V}$, and CUMS $+\mathrm{FLX}$ rats were tested (via the sucrose preference test) at 22 days and 44 days and sacrificed within $12 \mathrm{~h}$ of the last test. Rats in the CUMS + FLX + CUMS group were tested (via the sucrose preference test) at 22,44 , and 72 days, and sacrificed within $12 \mathrm{~h}$ of the last test. V: vehicle; CUMS: chronic unpredictable mild stress; FLX: fluoxetine; d: days; w: weeks.

$30 \mathrm{~min}, 4^{\circ} \mathrm{C}$ ) and boiled for $5 \mathrm{~min}$ in Laemmli buffer. The protein samples were separated at $70 \mathrm{~V}$ by $10 \%$ sodium dodecyl sulfate-polyacrylamide gel electrophoresis. The amount of protein loaded was $10 \mu \mathrm{g}$ for the total tau analysis and $40 \mu \mathrm{g}$ for phospho-tau (Ser356 and Thr231) analyses. After electrophoresis, the proteins were transferred to nitrocellulose membranes $(0.45-\mu \mathrm{m}$ pore size; Millipore Corp., USA) using a transfer unit run at $200 \mathrm{~mA}$ for $1 \mathrm{~h}$ for the analyses of phospho-tau and tau (Bio-Rad, USA). A molecular size marker (250-11.0 kDa range; Sigma, USA) was used to localize tau (50-80 kDa) and phospho-tau (Ser356, 46-80 kDa; Thr231, $60 \mathrm{kDa}$ ). The membranes were blocked for $2 \mathrm{~h}$ with $10 \%$ skim milk powder and incubated (overnight at $4^{\circ} \mathrm{C}$ ) with primary antibodies diluted in 5\% skim milk powder in Tris-buffered saline as follows: tau (Tau46, Cell Signaling, USA) at a 1:1000 dilution, phospho-tau (Ser356, Santa Cruz, USA) at a 1:100 dilution, phospho-tau (Thr231, Abcam, USA) at a 1:5000 dilution. The membranes were then incubated with horseradish peroxidase-conjugated secondary antibody (Promega, USA) for $1 \mathrm{~h}$. The protein band intensities were determined densitometrically using the CMIASWIN video imaging system (Bio-Rad). The densitometric values are reported as percentages of the control values. Glyceraldehyde phosphate dehydrogenase was used to normalize the quantification in Western blot experiments.

\section{Statistical analyses}

The results are reported as means $\pm S D$. Data were analyzed with a one-way ANOVA followed by the Bonferroni test for post hoc multiple comparisons. Statistically significant group differences were set at $\mathrm{P}<0.05$.

\section{Results}

\section{Sucrose preference tests}

We used the sucrose preference test to explore the effects of CUMS and re-exposure to CUMS on anhedonia in rats. Anhedonia is defined as "the decreased capacity to experience pleasure of any sort". The theoretical rationale for the chronic mild stress model is that this procedure simulates anhedonia, a loss of responsiveness to pleasant events, which is a core symptom of depression and the defining feature of melancholia (13).

Figure 2 shows the sucrose preference in the four groups of rats during the experimental period. As shown, 3 weeks of chronic stress induced a marked decrease in sucrose consumption in the stressed rats compared with the non-stressed rats (Figure 2A). After the first CUMS administration at 3 weeks, the percentage of sucrose consumed by the stressed animals was significantly lower than that of the non-stressed animals $[F(3,28)=8.937$, $\mathrm{P}<0.05]$. These results suggest that CUMS consistently induced an anhedonia.

Fluoxetine is a representative SSRI and was chosen as a reference compound for the validation of the chronic mild stress model (21). One-way ANOVA indicated that there were significant differences in the percentage of sucrose consumed among the four groups at the end of FLX treatment $[\mathrm{F}(3,28)=14.918, \mathrm{P}<0.05]$ (Figure 2B). Post hoc analysis found no differences between the control animals and the animals treated with FLX for 3 weeks. In contrast, the percentage of sucrose consumed was significantly different between the CUMS + V group and the other groups ( $\mathrm{P}<0.01$ for all comparisons). These results suggest that, after 3 weeks of FLX treatment, the sucrose preference changes of the stressed rats were reversed.

After drug washout, the CUMS + FLX + CUMS group received an additional exposure to CUMS. We compared the results of CUMS $+\mathrm{V}$ at 44 days and the results of CUMS + FLX + CUMS at 72 days (Figure 2C). The animals that were re-treated with CUMS consumed significantly less sucrose solution $(P<0.05)$ than the $\mathrm{CUMS}+\mathrm{V}$ rats (Figure $2 \mathrm{C}$ ). This suggests that, after re-exposure to CUMS, the anhedonia in the CUMS+ $F L X+C U M S$ rats became more intense. 

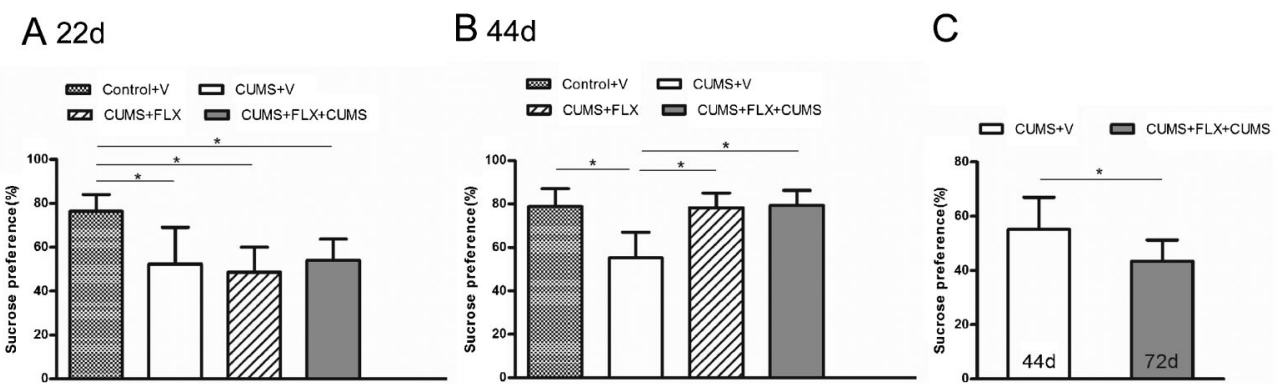

Figure 2. A, Effects of chronic unpredictable mild stress (CUMS) on sucrose preference in rats. Prior to each test, rats were waterdeprived for $12 \mathrm{~h}$. They were then exposed to both a $1 \%$ sucrose solution and tap water. $B$, Effects of fluoxetine (FLX) on sucrose preference in rats. $C$, Effects of re-exposure to CUMS on sucrose preference in rats. Data are reported as means $\pm S D$. ${ }^{*} P<0.01$ (Panels $A$ and $B$ ) and ${ }^{*} \mathrm{P}<0.05$ (Panel $C$ ) (one-way ANOVA followed by the Bonferroni test). V: vehicle; d: days.

Effects of CUMS, FLX, and re-exposure to CUMS on tau and phospho-tau (Thr231 and Ser356) protein levels in the rat hippocampus and frontal cortex

Acute stress has been reported to induce robust hyperphosphorylation of tau in the frontal cortex and hippocampus of animals (22). Furthermore, dysfunction of the frontal cortex has been implicated in the neurobiology of depressive illnesses (23). Thus, to investigate whether depression could promote detrimental tau hyperphosphorylation, we quantified the levels of phospho-tau in both the hippocampus and the frontal cortex of chronicstressed animals. According to the product information, the tau46 antibody recognizes hippocampal tau regardless of its phosphorylation state. The p-tau (Ser356) and p-tau (Thr231) antibodies stain only the phosphorylated form of rat tau, and only when it is phosphorylated at serine 356 and threonine 231, respectively. The effects of stress and FLX on phospho-tau protein levels in the hippocampus and frontal cortex are reported in Figure $3 \mathrm{~A}$ and $B$, respectively.

One-way ANOVA revealed that, in the hippocampus and frontal cortex, the staining of tau by tau46 was not significantly altered after stress or re-exposure to stress (Figure 3A and B, first band), but the levels of phosphotau (Ser356 and Thr231) were significantly altered after stress and re-exposure to stress (Figure $3 A$ and $B$, middle and bottom panels.

In the hippocampus, densitometric analyses showed that phospho-tau (Ser356) protein levels increased significantly $(152 \pm 12 \%, P<0.01)$ in CUMS $+V$ rats subjected to stress once, and also increased significantly $(221 \pm 14 \%$, $\mathrm{P}<0.01)$ following re-exposure to stress in the CUMS + FLX + CUMS group, compared with the Control + V group. Densitometric analyses of phospho-tau (Thr231) protein levels showed a significant $166 \pm 14 \%$ increase $(P<0.01)$ in $\mathrm{CUMS}+\mathrm{V}$ rats subjected to stress once, and a significant $302 \pm 17 \%$ increase $(P<0.01)$ in the CUMS + FLX + CUMS group following re-exposure to stress, compared with the Control $+\mathrm{V}$ group. Post hoc analysis showed that the densitometric values of phospho-tau
(Ser356 and Thr231) protein levels in the hippocampus were higher in the CUMS + FLX + CUMS group compared with the CUMS $+\mathrm{V}$ group $(\mathrm{P}<0.01$ for both, Bonferroni test). However, phospho-tau (Ser356 and Thr231) protein levels in the CUMS +FLX rats did not differ significantly from the Control $+V$ group $(P>0.05$ for both).

The results in the frontal cortex were very similar to those in the hippocampus. CUMS also increased the levels of phospho-tau (Ser356 and Thr231), and rats that received an additional CUMS exposure after recovering from CUMS-induced depression (i.e., anhedonia) showed higher phospho-tau (Ser356 and Thr231) protein levels. Details of the main results are given in Table 1.

\section{Discussion}

The present study revealed elevation of the levels of phosphorylated tau in the hippocampus and frontal cortex of depressive animals, which may indicate a link between depression and $A D$. In $A D$ brains, tau moves from the axons to the somatodendritic compartment of neurons, where it forms hyperphosphorylated, filamentous aggregates described as neurofibrillary tangles. Postmortem studies report greater neurofibrillary tangle pathology in $A D$ patients with a lifetime history of depression compared with those without such a history (8), and more severe cortical neurofibrillary tangle pathology is found in the brains of $A D$ subjects who suffered from comorbid depression (9). The association between depression and $A D$ may emerge from the impact that depression and depressive symptoms have on the hypothalamicpituitary-adrenal axis, resulting in impaired negative feedback and chronic elevation of adrenal glucocorticoids (24). In turn, in animal studies, glucocorticoids have been reported to promote amyloid precursor protein expression and tau accumulation $(25,26)$. Thus, longer glucocorticoid exposure augments tau accumulation and might additionally lead to pathological alterations in tau, including alterations in its phosphorylation state. Based upon these studies, we propose that depression leads to elevated tau 


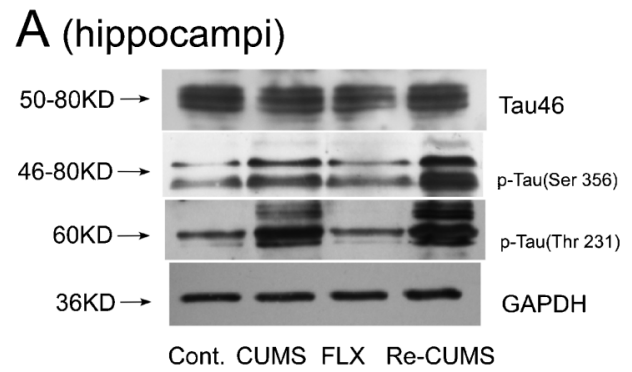

p-Tau(Ser356)

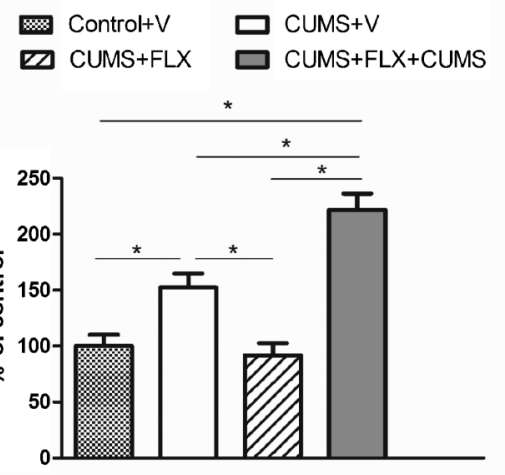

p-Tau(Thr231)

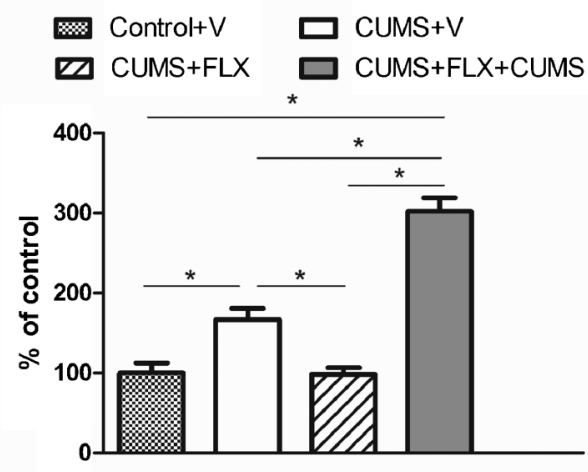

\section{B (frontal cortex)}

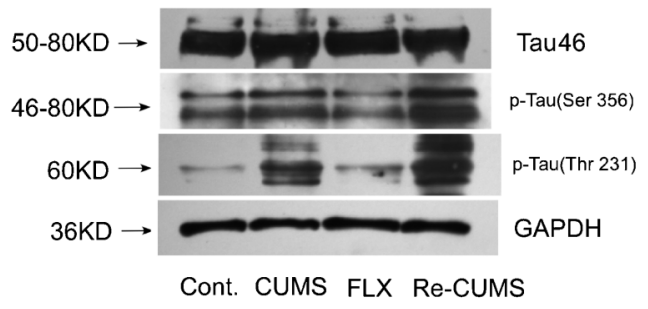

p-Tau(Ser356)

$\begin{array}{ll}\otimes \text { Control+V } & \square \text { CUMS+V } \\ \square \text { CUMS+FLX } & \square \text { CUMS+FLX+CUMS }\end{array}$

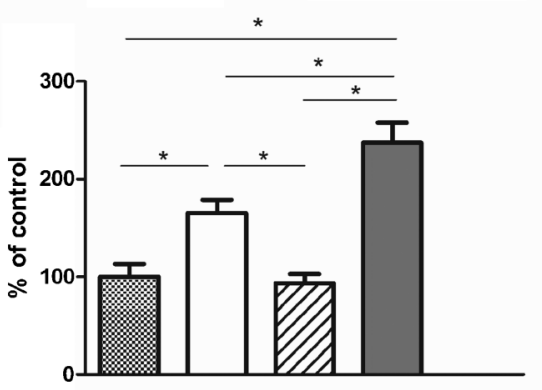

p-Tau(Thr231)

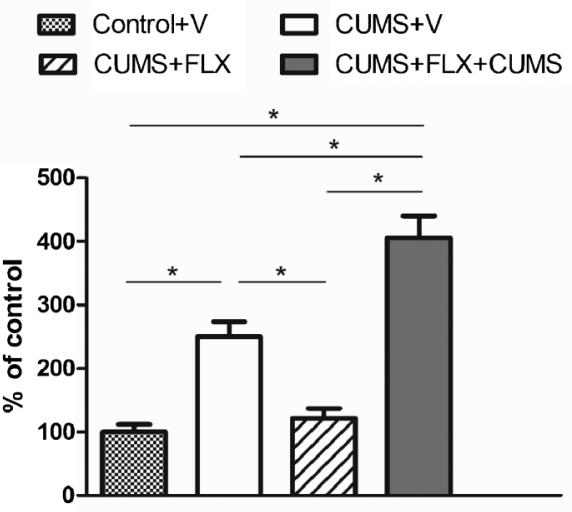

Figure 3. Effects of chronic unpredictable mild stress (CUMS), fluoxetine (FLX), and re-exposure to CUMS on the levels of tau and phospho-tau in the hippocampi and the frontal cortex of rats. GAPDH was used to normalize the quantification in Western blot experiments. Representative Western blot bands of the hippocampi $(A)$ and the frontal cortex $(B)$ and densitometric analyses of the bands for p-Tau (Ser356) and p-Tau (Thr231) ratio of the hippocampi, and p-Tau (Ser356) and p-Tau (Thr231) ratio of the frontal cortex. Cont: Control + vehicle (V); CUMS: CUMS +V; FLX: CUMS +FLX; Re-CUMS: CUMS +FLX + CUMS. Data are reported as means $\pm S D$. ${ }^{*} P<0.01$ (one-way ANOVA followed by the Bonferroni test).

phosphorylation, which is a key pathological agent in the development of $A D$, and that glucocorticoids may play a critical role in tau phosphorylation. Thus, our findings may offer an insight into the mechanisms behind the link between depression and $A D$ that is suggested by epidemiologic data showing prior depression to be an etiologic risk factor for AD. However, the currently available data are insufficient to support an exclusive association between depression and AD per se. The precise mechanism remains unclear and requires further research. 
Table 1. Main results of the present study.

\begin{tabular}{lcccccc}
\hline & \multicolumn{2}{c}{$\mathrm{p}$-Tau (Ser356) } & & \multicolumn{2}{c}{$\mathrm{p}$-Tau (Thr231) } \\
\cline { 2 - 3 } \cline { 6 - 6 } & Hippocampus & Frontal cortex & & Hippocampus & Frontal cortex \\
\hline CUMS & $\uparrow$ & $\uparrow$ & & $\uparrow$ & $\uparrow$ \\
FLX & $\downarrow$ & $\downarrow$ & & $\downarrow$ & $\downarrow$ \\
Re-exposure to CUMS & $\uparrow \uparrow$ & $\uparrow \uparrow$ & & $\uparrow \uparrow$ & $\uparrow \uparrow$ \\
\hline
\end{tabular}

CUMS: chronic unpredictable mild stress; FLX: fluoxetine. $\uparrow:$ increased; $\downarrow$ : decreased; $\uparrow \uparrow:$ increased more intensely.

The alterations in tau phosphorylation observed here suggest an additional mechanism, by which depression decreases microtubule dynamics. In a previous study (27), we showed that CUMS induced a decrease in TyrTub and an increase in Acet-Tub protein levels, which together suggest decreased microtubule dynamics. The neuronal hyperphosphorylation of tau is a well-known cytoskeletal change related to decreased microtubule dynamics (28). Changes in the dynamic status of the microtubule cytoskeleton are directly related to neuronal morphology (11). The intrinsic dynamic instability of microtubules is fundamental in regulating the axonal trafficking of vesicles (10). Hyperphosphorylated tau is prone to form aggregates, which may block the intracellular trafficking of neurotrophics and other functional proteins. This may result in a loss or decline in axonal or dendritic transport in the neurons (29). Neurons with tau hyperphosphorylation are considered "sick" because these neurons are no longer competent for axonal transport (30), an early marker of neurodegeneration in neurodegenerative diseases, including AD (30).

The severity of depression has been found to be an important predictor of the risk of dementia. One longitudinal study reported that depression led to as much as a 2-fold increased risk of a diagnosis of dementia over 7 years compared to controls without dysthymia or depression and that there was a "dose-effect" wherein depression resulted in a higher risk than dysthymia (31). Another study demonstrated a strong association between the number of depressive episodes (i.e., recurrent depression) and the risk of dementia over a median follow-up time of 24 years, suggesting a cumulative, dose-dependent relationship of depression episodes and risk of dementia (32). Our study showed that, after re-exposure to CUMS, sucrose consumption in the CUMS + FLX + CUMS group was further impaired and was associated with even higher phospho-tau protein levels. We cautiously interpret our findings as a partial explanation of the evidence from clinical studies showing that patients with recurrent depression and more severe episodes have a higher risk of dementia(s) and AD than those with a single episode or multiple, less severe episodes. However, more research is required to determine the mechanisms.

We chose to investigate two phosphorylation sites
(Thr231 and Ser356). Phosphorylation of tau at Thr231 plays a critical role in regulating tau's ability to bind and stabilize microtubules (33), and increased phosphorylation of Thr231 has been proposed as an early event in AD pathogenesis (34). Ser356 is located in the tau microtubule-binding domain, and hyperphosphorylation at this site is thought to cause a greater decrease in tau's promotion of microtubule assembly and stabilization than hyperphosphorylation at other sites (7).

Antidepressants are a rational complementary therapy for the treatment of $A D$ (35). In fact, long-term treatment with SSRIs including FLX improves memory function in AD patients (36). Long-term paroxetine treatment significantly reduces tau immunoreactivity in the hippocampus (37). The results of our study, which showed that FLX decreased tau phosphorylation in the hippocampus and frontal cortex, are consistent with previous paroxetine studies. The possible mechanisms of these antidepressant effects on $A D$ include stimulating neurogenesis, increasing long-term potentiation, anti-inflammatory activity, and inhibiting $\mathrm{N}$ methyl-D-aspartate receptors $(30,38)$. The decrease in tau pathology by FLX that was observed in our study may offer another clue to the understanding of antidepressant action in AD. However, the mechanisms by which paroxetine and FLX suppress tau pathology remain unknown. The antidepressant actions of paroxetine and FLX are thought to be mediated by the inhibition of serotonin reuptake, resulting in enhanced serotonergic signaling and up-regulation of brain-derived neurotrophic factor gene expression (39). Further research is required to gain understanding of these changes, for example, by testing specific serotonin receptor agonists or antagonists.

In conclusion, our results suggest that elevated phosphorylated tau levels following chronic stress may contribute to the mechanisms of depression as an etiologic risk factor for $A D$, as suggested by epidemiologic data. The efficacy of FLX in modifying tau phosphorylation may offer another clue to why antidepressants are a rational complementary therapy for $A D$.

\section{Acknowledgments}

We sincerely thank the Key Laboratory of HuBei Province for Digestive System Diseases for its support. 


\section{References}

1. Byers AL, Yaffe K. Depression and risk of developing dementia. Nat Rev Neurol 2011; 7: 323-331, doi: 10.1038/ nrneurol.2011.60.

2. Geerlings MI, den Heijer $T$, Koudstaal PJ, Hofman $A$, Breteler MM. History of depression, depressive symptoms, and medial temporal lobe atrophy and the risk of Alzheimer disease. Neurology 2008; 70: 1258-1264, doi: 10.1212/ 01.wnl.0000308937.30473.d1.

3. Barnes DE, Yaffe K, Byers AL, McCormick M, Schaefer C, Whitmer RA. Midlife vs late-life depressive symptoms and risk of dementia: differential effects for Alzheimer disease and vascular dementia. Arch Gen Psychiatry 2012; 69: 493498, doi: 10.1001/archgenpsychiatry.2011.1481.

4. Green RC, Cupples LA, Kurz A, Auerbach S, Go R, Sadovnick D, et al. Depression as a risk factor for Alzheimer disease: the MIRAGE Study. Arch Neurol 2003; 60: 753-759, doi: 10.1001/archneur.60.5.753.

5. Wuwongse S, Chang RC, Law AC. The putative neurodegenerative links between depression and Alzheimer's disease. Prog Neurobiol 2010; 91: 362-375, doi: 10.1016/ j.pneurobio.2010.04.005.

6. Butters MA, Young JB, Lopez O, Aizenstein HJ, Mulsant $\mathrm{BH}$, Reynolds $\mathrm{CF}$ III, et al. Pathways linking late-life depression to persistent cognitive impairment and dementia. Dialogues Clin Neurosci 2008; 10: 345-357.

7. Wang JZ, Liu F. Microtubule-associated protein tau in development, degeneration and protection of neurons. Prog Neurobiol 2008; 85: 148-175, doi: 10.1016/j.pneurobio. 2008.03.002.

8. Rapp MA, Schnaider-Beeri M, Grossman HT, Sano M, Perl DP, Purohit DP, et al. Increased hippocampal plaques and tangles in patients with Alzheimer disease with a lifetime history of major depression. Arch Gen Psychiatry 2006; 63: 161-167, doi: 10.1001/archpsyc.63.2.161.

9. Rapp MA, Schnaider-Beeri M, Purohit DP, Perl DP, Haroutunian V, Sano M. Increased neurofibrillary tangles in patients with Alzheimer disease with comorbid depression. Am J Geriatr Psychiatry 2008; 16: 168-174, doi: 10.1097/JGP.0b013e31816029ec.

10. Bianchi M, Hagan JJ, Heidbreder CA. Neuronal plasticity, stress and depression: involvement of the cytoskeletal microtubular system? Curr Drug Targets CNS Neurol Disord 2005; 4: 597-611, doi: 10.2174/156800705774322012.

11. Cuadrado-Tejedor M, Ricobaraza A, Del Rio J, Frechilla D, Franco R, Perez-Mediavilla A, et al. Chronic mild stress in mice promotes cognitive impairment and CDK5-dependent tau hyperphosphorylation. Behav Brain Res 2011; 220: 338343, doi: 10.1016/j.bbr.2011.01.005.

12. Lee AL, Ogle WO, Sapolsky RM. Stress and depression: possible links to neuron death in the hippocampus. Bipolar Disord 2002; 4: 117-128, doi: 10.1034/j.1399-5618.2002. 01144.x

13. Willner $\mathrm{P}$. Chronic mild stress (CMS) revisited: consistency and behavioural-neurobiological concordance in the effects of CMS. Neuropsychobiology 2005; 52: 90-110, doi: 10.1159/000087097.

14. Surget A, Saxe M, Leman S, Ibarguen-Vargas Y, Chalon S, Griebel G, et al. Drug-dependent requirement of hippocampal neurogenesis in a model of depression and of antidepressant reversal. Biol Psychiatry 2008; 64: 293301, doi: 10.1016/j.biopsych.2008.02.022.

15. Luo DD, An SC, Zhang X. Involvement of hippocampal serotonin and neuropeptide $\mathrm{Y}$ in depression induced by chronic unpredicted mild stress. Brain Res Bull 2008; 77: 812, doi: 10.1016/j.brainresbull.2008.05.010.

16. Moreau JL, Jenck F, Martin JR, Mortas P, Haefely WE. Antidepressant treatment prevents chronic unpredictable mild stress-induced anhedonia as assessed by ventral tegmentum self-stimulation behavior in rats. Eur Neuropsychopharmacol 1992; 2: 43-49, doi: 10.1016/0924977X(92)90035-7.

17. Willner P, Towell A, Sampson D, Sophokleous S, Muscat R. Reduction of sucrose preference by chronic unpredictable mild stress, and its restoration by a tricyclic antidepressant. Psychopharmacology 1987; 93: 358-364, doi: 10.1007/BF00187257.

18. Caccia S, Cappi M, Fracasso C, Garattini S. Influence of dose and route of administration on the kinetics of fluoxetine and its metabolite norfluoxetine in the rat. Psychopharmacology 1990; 100: 509-514, doi: 10.1007/BF02244004.

19. Anthony JP, Sexton TJ, Neumaier JF. Antidepressantinduced regulation of $5-\mathrm{HT}(1 \mathrm{~b}) \mathrm{mRNA}$ in rat dorsal raphe nucleus reverses rapidly after drug discontinuation. $J$ Neurosci Res 2000; 61: 82-87, doi: 10.1002/10974547(20000701)61:1<82::AID-JNR10>3.0.CO;2-E.

20. Bianchi M, Heidbreder C, Crespi F. Cytoskeletal changes in the hippocampus following restraint stress: role of serotonin and microtubules. Synapse 2003; 49: 188-194, doi: 10. 1002/syn.10230.

21. Roy M, David N, Cueva M, Giorgetti M. A study of the involvement of melanin-concentrating hormone receptor 1 (MCHR1) in murine models of depression. Biol Psychiatry 2007; 61: 174-180, doi: 10.1016/j.biopsych.2006.03.076.

22. Yan J, Sun XB, Wang $H Q$, Zhao $H$, Zhao XY, Xu YX, et al. Chronic restraint stress alters the expression and distribution of phosphorylated tau and MAP2 in cortex and hippocampus of rat brain. Brain Res 2010; 1347: 132-141, doi: 10.1016/j.brainres.2010.05.074

23. Anisman H, Merali Z, Hayley S. Neurotransmitter, peptide and cytokine processes in relation to depressive disorder: comorbidity between depression and neurodegenerative disorders. Prog Neurobiol 2008; 85: 1-74, doi: 10.1016/ j.pneurobio.2008.01.004.

24. Sapolsky RM, Krey LC, McEwen BS. The neuroendocrinology of stress and aging: the glucocorticoid cascade hypothesis. Endocr Rev 1986; 7: 284-301, doi: 10.1210/ edrv-7-3-284.

25. Budas G, Coughlan CM, Seckl JR, Breen KC. The effect of corticosteroids on amyloid beta precursor protein/amyloid precursor-like protein expression and processing in vivo. Neurosci Lett 1999; 276: 61-64, doi: 10.1016/S03043940(99)00790-9.

26. Green KN, Billings LM, Roozendaal B, McGaugh JL, LaFerla FM. Glucocorticoids increase amyloid-beta and tau pathology in a mouse model of Alzheimer's disease. $J$ Neurosci 2006; 26: 9047-9056, doi: 10.1523/JNEUROSCI. 2797-06.2006.

27. Yang C, Wang G, Wang H, Liu Z, Wang X. Cytoskeletal alterations in rat hippocampus following chronic unpredictable 
mild stress and re-exposure to acute and chronic unpredictable mild stress. Behav Brain Res 2009; 205: 518-524, doi: 10.1016/j.bbr.2009.08.008.

28. Arendt T, Stieler J, Strijkstra AM, Hut RA, Rudiger J, Van der Zee EA, et al. Reversible paired helical filament-like phosphorylation of tau is an adaptive process associated with neuronal plasticity in hibernating animals. $J$ Neurosci 2003; 23: 6972-6981.

29. Salehi A, Delcroix JD, Mobley WC. Traffic at the intersection of neurotrophic factor signaling and neurodegeneration. Trends Neurosci 2003; 26: 73-80, doi: 10.1016/S0166-2236 (02)00038-3.

30. Yang Y, Yang XF, Wang YP, Tian Q, Wang XC, Li HL, et al. Inhibition of protein phosphatases induces transport deficits and axonopathy. J Neurochem 2007; 102: 878-886, doi: 10.1111/j.1471-4159.2007.04603.x

31. Byers AL, Covinsky KE, Barnes DE, Yaffe K. Dysthymia and depression increase risk of dementia and mortality among older veterans. Am J Geriatr Psychiatry 2012; 20: 664-672, doi: 10.1097/JGP.0b013e31822001c1.

32. Dotson VM, Beydoun MA, Zonderman AB. Recurrent depressive symptoms and the incidence of dementia and mild cognitive impairment. Neurology 2010; 75: 27-34, doi: 10.1212/WNL.0b013e3181e62124

33. Cho JH, Johnson GV. Primed phosphorylation of tau at Thr231 by glycogen synthase kinase 3beta (GSK3beta) plays a critical role in regulating tau's ability to bind and stabilize microtubules. J Neurochem 2004; 88: 349-358, doi: 10.1111/j.1471-4159.2004.02155.x.

34. Buerger K, Teipel SJ, Zinkowski R, Blennow K, Arai $H$, Engel R, et al. CSF tau protein phosphorylated at threonine 231 correlates with cognitive decline in $\mathrm{MCl}$ subjects. Neurology 2002; 59: 627-629, doi: 10.1212/WNL.59.4.627.

35. Aboukhatwa M, Dosanjh L, Luo Y. Antidepressants are a rational complementary therapy for the treatment of Alzheimer's disease. Mol Neurodegener 2010; 5: 10, doi: 10.1186/1750-1326-5-10.

36. Mowla A, Mosavinasab M, Haghshenas $H$, Borhani HA Does serotonin augmentation have any effect on cognition and activities of daily living in Alzheimer's dementia? A double-blind, placebo-controlled clinical trial. J Clin Psychopharmacol 2007; 27: 484-487, doi: 10.1097/jcp.0b 013e31814b98c1.

37. Nelson RL, Guo Z, Halagappa VM, Pearson M, Gray AJ, Matsuoka $Y$, et al. Prophylactic treatment with paroxetine ameliorates behavioral deficits and retards the development of amyloid and tau pathologies in 3xTgAD mice. Exp Neurol 2007; 205: 166-176, doi: 10.1016/j.expneurol.2007.01.037.

38. Liu D, Wang Z, Liu S, Wang F, Zhao S, Hao A. Antiinflammatory effects of fluoxetine in lipopolysaccharide(LPS)stimulated microglial cells. Neuropharmacology 2011; 61: 592599, doi: 10.1016/j.neuropharm.2011.04.033.

39. Mattson MP, Maudsley S, Martin B. BDNF and 5-HT: a dynamic duo in age-related neuronal plasticity and neurodegenerative disorders. Trends Neurosci 2004; 27: 589594, doi: 10.1016/j.tins.2004.08.001. 